\title{
Transcriptome Analysis and Metabolic Profiling of Anthocyanins Accumulation in Loropetalum Chinense var. Rubrum With Different Colors of Leaf
}

\section{Xia Zhang}

Hunan Agricultural University

\section{Li Zhang}

Hunan Agricultural University

Damao Zhang

Hunan Agricultural University

\section{Dingding Su}

Peking University

\section{Weidong Li}

Hunan Institute of Science and Technology

\section{Xiangfei Wang}

Hunan Agricultural University

\section{Qianru Chen}

Hunan Agricultural University

\section{Wenqi Cai}

Hunan Agricultural University

Lu Xu

Hunan Agricultural University

\section{Fuxiang Cao}

Hunan Agricultural University

\section{Dongling Zhang}

Georgia State University

\section{Xiaoying Yu}

Hunan Agricultural University

Yanlin Li ( $D$ liyanlin@hunau.edu.cn )

Hunan Agricultural University https://orcid.org/0000-0002-1114-6940

\section{Research Article}

Keywords: leaf color, anthocyanin, synthesis pathway, Loropetalum chinense var. rubrum 
Posted Date: February 28th, 2022

DOI: https://doi.org/10.21203/rs.3.rs-960260/v1

License: (c) (i) This work is licensed under a Creative Commons Attribution 4.0 International License. Read Full License 


\section{Abstract}

Background: Loropetalum chinense var. rubrum is a typical colorful ornamental and medicinal plant. However, the coloration information and potential colorful molecular mechanisms of its leaves remain unclear. We analyzed structural and regulatory genes for flavonoid metabolites and anthocyanin biosynthesis in 3 kinds color of leaves $₫ \mathrm{PL}$, pink leaves; $\mathrm{ML}$, middle color leaves; $\mathrm{GL}$, green leaves) of $L$. chinense var. rubrum.

Results: Metabolic detection to the samples showed that there were significant differences in the metabolites accumulated in different color leaves. Meanwhile, transcriptome indicated that DFR, ANR and UFGT genes in L. chinense var. rubrum might be one of the reasons for the accumulation of anthocyanin in ML and GL. We also found that several key members of the MYB, BHLH, and WD40 gene families played important roles in regulating the accumulation of flavonoids in plants, which were considered to be the main drivers of transcriptional changes in different colors of $L$. chinense var. rubrum leaves. Our findings provided a comprehensive data set for the metabolism and transcription of L. chinense var. rubrum as well as candidate structures and transcription factors information related to leaf pigmentation.

Conclusions: The conclusion would provide useful information for elucidating the formation of leaf color and anthocyanin accumulation in L. chinense var. rubrum $\rrbracket$ which could also explain the color variation in its leaves.

\section{Background}

Loropetalum chinense var. rubrum belongs to the Hamamelidaceous (witch-hazel family)[1] and is mainly distributed in the south of the middle Yangtze River to the north of the Tropic of Cancer in China. It originated from Hunan Province and plays an important role in the landscape[2]. L. chinense. var. rubrum is an evergreen plant with a beautiful habit and bright colorful foliage. The plant is also a traditional Chinese medicine and its leaves, flowers, and roots can be used for treating cough, burn, uterine bleeding, traumatic bleeding, abdominal pain, diarrhea, etc. [3]. Recently, the plant has gained a lot of popularity and is widely cultivated for its ornamental and medicinal importance.

The leaf color of L. chinense var. rubrum depends on a mixture of chlorophyll, carotenoids, and flavonoids. Flavonoids are the decisive pigment presented in most plant colors, which provide blue, pink, yellow, purple, and red for plants[4, 5]. Among these, flavonoids are comprised of chalcones, flavone, flavanol, and anthocyanins[6]. It is generally believed that the color abundance of plant foliage is caused by the content and composition of anthocyanins. In many fruits, flowers, and vegetables, the appearance of color was determined by the accumulation of anthocyanins, such as Vaccinium corymbosum [7], Morella rubra [8], Centaurea cyanus [9], Primula vulgaris[10], Onion(Allium cepa L)[11]. Anthocyanins could reduce the stress of cold, drought, and pests on plants [12-14], and it is also beneficial to human health as it was proved to make a great contribution to anti-oxidation, anti-cancer, anti-bacterial, cardiovascular, and neurodegenerative disease protection $[15,16]$. The variations of anthocyanins in 
higher plants are complex, generally divided into six categories: Cyanidin, Pelargonidin, Delphinidin, Peonidin, Petunidin, and Malvidin[17].

The anthocyanin synthesis followed the flavonoid and anthocyanin biosynthesis pathway, under the phenylpropanoid pathway[18]. The formation of these anthocyanins required phenylpropane as a substrate under the involvement of a series of enzymes, such as PAL, C4H, $4 \mathrm{CL}, \mathrm{CHS}, \mathrm{CHI}, \mathrm{F} 3 \mathrm{H}, \mathrm{CYP75A}$, DFR, ANS and UFGT[19]. The type of accumulated anthocyanins was identified by $\mathrm{F} 3 \mathrm{H}$ and $\mathrm{F}^{\prime} \mathrm{5}^{\prime} \mathrm{H}[20-$ 22]. UGFT is the final enzyme gene for anthocyanin to be transformed into a stable state[23].

Anthocyanin accumulation can also activate or inhibit the spatiotemporal expression of structural genes through transcription factors. Such as MYB, bHLH, and WD40 proteins [24]. MYB is one of the most abundant transcription factors in higher plants, which is involved in the regulation of secondary metabolism, cell morphogenesis and differentiation, signal transduction, and stress response[25-28]. bHLH transcription factors regulated anthocyanin synthesis by binding with MYB transcription factors /WD 40 proteins or forming an MBW protein complex with MYB transcription factors and WD 40 proteins[29-31].

The secondary metabolites of $L$. chinense. var. rubrum is mainly flavonoids, which is related to the formation of leaf color. The accumulation of these compounds in the leaves is different, which is the main reason for the different leaf colors. However, due to the limitation of genetic background, in-depth research on the molecular mechanism and genetic improvement of leaf coloration of L. chinense. var. rubrum is hindered. Studying the accumulation of flavonoids in $L$. chinense. var. rubrum is of great significance for studying the molecular mechanism of leaf coloration.

In this study, the process of flavonoids biosynthesis was identified by analyzing the transcriptome and metabolites of different $L$. chinense. var. rubrum leaf colors. The gene expression and metabolites related to leaf color formation were revealed. The findings should greatly enhance the better understanding of the formation mechanism of Loropetalum leaves.

\section{Results}

\section{Determination of photosynthetic and anthocyanin content}

To elucidate the mechanism of biosynthesis of pigments in three-leaf colors of L. chinense. var. rubrum, the GL, ML, and PL were chosen for this study (Fig. 1). The contents of chlorophyll and anthocyanin in these three leaf colors were distinct. Compared to the GL and ML, PL had relatively lower levels of carotenoid and total chlorophyll but higher anthocyanin accumulation, which indicated that anthocyanin might be the main pink-colored material in leaves.

\section{Differential metabolites from different leaves}

Since anthocyanin content is a determinant for leaf color change in the plant, we profiled the metabolome of the leaf samples of GL, PL, and ML using the LC-MS/MS analysis and annotated it in the MS2 
database. A total of 207 compounds were detected in the L. chinense. var. rubrum leaf, which could be grouped into eight classes, including Proanthocyanins, Polyphenol, Isoflavone, Flavonol, Flavanone, Anthocyanins, Flavonoid, Flavone, etc. (Additional file 1) All metabolites were analyzed by principal component analysis and hierarchical cluster analysis to determine the influence of leaf-color change on the change of metabolites. (Fig. 2A) [32]. A Fold change ( $\geq 2$ or $\leq 0.5)$ among the metabolites with a VIP $>1$ was used as an identification criterion. In all group comparisons, a total of 11 metabolites showed significant differences in each combination. (Fig. 2B). There were 35 differential metabolites (31 upregulated and 4 down-regulated) between $\mathrm{GL}$ and PL (Fig. 2C), 37(33 up-regulated and 4 down-regulated) between GL and ML (Fig. 2D). Two cluster heat maps of 46 flavonoid compounds were obtained. (Fig. 2E, F). Interestingly, all the differentially accumulated anthocyanins were up-accumulated in ML and PL samples.

\section{Differential metabolic pathways in different leaves}

The enrichment of differential metabolites in the metabolic pathway by the KEGG database helps us to understand the changes in the mechanisms of metabolic pathways in different leaf colors. (Additional file 2). Notably, different metabolites were enriched in the KEGG metabolic pathway. Compared with GL and $\mathrm{ML}, \mathrm{PL}$ was mainly involved in biosynthesis, including flavonoid biosynthesis and anthocyanin biosynthesis.

\section{Transcriptome de novo assembly, annotation}

Three standardized cDNA libraries were constructed from RNA from GL, ML, and PL. After the CDNA library was cleaned and characterized, $186,694,570,149,946,386$ and 123,143,062 clean reads were obtained, respectively. More than $98.09 \%$ of them have Q20(an error probability of $0.02 \%$ ) for GL, PL and ML were $97.9 \%, 97.48 \%$. The GC contents were approximately $43.73 \% 43.84 \%$, and $43.98 \%$ for $\mathrm{GL}$, PL, and ML (Additional file 3 ). These clean reads were assembled into 231,810 unigenes with lengths ranging from 65 to 21,135 bp (average length $1271 \mathrm{bp}$ ) and an N50 of $2608 \mathrm{bp}$. There were 110,962 unigenes $(47.87 \%)$ with a length of $<500 \mathrm{bp} ; 31,726$ unigenes (13.69\%) with a length of $500-1000 \mathrm{bp} ; 38,050$ $(16.41 \%)$ unigenes with a length of $1000-2000$ bp; 23,499 unigenes $(10.14 \%)$ with a length of $2000-3000$ $\mathrm{bp}$, and the remaining 27,573 unigenes (11.89\%) with a length of $>3000 \mathrm{bp}$ (Additional file 4). The sequencing quality was able to cover the vast majority of expressed genes in $G L, M L$, and $P L$, which provided the foundation for further analysis.

For comprehensive annotation, all the assembled unigene sequences were matched against NR, SwissProt, KEGG, KOG, GO, NT and Pfam databases produced

118,518(17.88\%);96,572(14.57\%);115,058(17.36\%);75,254(11.36\%);84,662(12.77\%);88,001(13.28\%) and $84,662(12.77 \%)$ respectively (Additional file 5).

With GO classification, 391,895 matched unigenes were classified into 3 major categories: biological process, molecular function, and cellular component (Additional file $6 \mathrm{~A}$ ). In the group of biological processes, the majority of unigenes involved in the metabolic process $(43,655)$ and cellular process 
$(41,359)$; in the cellular components group, the cell $(21,255)$ and cell part $(21,255)$; and the molecular function group, binding $(47,320)$ and catalytic activity $(41,565)$.

The annotated unigenes were assigned to the KEGG database for the identification of the biochemical pathways (Additional file 6B). A total of 72,251 unigenes were divided into 46 metabolic pathways under six main branches: cellular processes $(6,659)$, environmental information processing $(5,400)$, genetic information processing $(11,381)$, human diseases $(13,719)$, metabolism $(25,260)$, and organismal systems $(9,832)$. The top ten metabolic genes in subclasses included the following: signal transduction, translation, carbohydrate metabolism, global and overview maps, folding sorting and degradation, transport and catabolism, viral, energy metabolism, amino acid metabolism, and overview.

In total, pathways related to cellular processes and environmental information processing were also well represented by unigenes from $L$. chinense. var. rubrum. This result should play an important role in our metabolic pathway research in $L$. chinense. var. rubrum leaves.

\section{Analysis of the differential expression genes in different colors}

We applied the standard $|\mathrm{Log} 2 \mathrm{FC}| \geq 2$ to make all pairwise comparisons of the different expressions between GL, PL, and ML leaf colors. We identified 6743 significant DEGs between the GL and ML and 1636 significant DEGs between the GL and PL. With GL set as control, the numbers of up-regulated and down-regulated genes in ML were 2855 and 3888, respectively. 910 DEGs were up-regulated and 726 DEGs were down-regulated between GL and PL (Fig. 3A, B).

Go allocation was applied for predicting the function of gene expression that is involved in the flavonoid biosynthesis pathway. The most important enrichment Go terms are selected and displayed in figure (Additional file 7), and the most significant enrichment was found in the correlation between GL and $\mathrm{ML}$, such as metabolic process, organic substance, metabolic process, primary metabolic process, and cellular metabolic process. Oxidation-reduction process, single-organic metabolic process, oxidoreductase activity and catalytic are significant between GL and PL.

To further investigate the biochemical pathways of these DEGs, all of which were mapped to terms in the KEGG database, which was a pathway-related database that could help better understand specific processes, gene functions, and gene interactions at the transcriptomic level[33]. There were 591 (GL vs $\mathrm{ML}$ ) and 247 (GL vs PL) DEGs mapped onto the top 20 enriched KEGG pathways in the L. chinense. var. rubrum transcriptomes from different colors of leaves. In these samples, flavone and flavanol biosynthesis(ko00942) and Flavonoid biosynthesis (ko00941) were closely related to anthocyanin synthesis (Additional file 8).

The focus of this study is the difference in anthocyanin accumulation among different leaf colors. Previous anthocyanin determination results showed that the anthocyanin content in GL was significantly lower than in ML and PL (Fig. 1A), which could prove the color substance of L. chinense var. rubrum leaves were anthocyanin. Therefore, we focused on the metabolic pathways related to anthocyanin 
synthesis. Genes enriched in this pathway were 2 ANR (EC:1.3.1.77; ANR1467 and ANR1398); 4 CYP75A (EC:1.14.14.81; CYP75A1815 CYP75A2846, CYP75A2909 and CYP75A1716); 1 DFR (EC:1.1.1.219; DFR92899), and 2 UFGT (EC:2.4.1.115; UFGT1876 and UFGT3273).In addition to the structural genes, MYB, bHLH and WD40 great influence on anthocyanin biosynthesis[34]. Because the leaves appear to be of different colors and have different anthocyanins, we will focus on the transcription factors and select 3 MYB, 3 bHLH, and 3 WD40 transcription factors.

\section{qRT-PCR validation transcriptome results}

To verify the expression levels of structural genes and transcription factors related to anthocyanin synthesis in $L$. chinense var. rubrum, $\beta$-actin was selected as the reference gene. The results showed that the expression was similar to what had been obtained by RNA-Seq (Fig. 4A). ANR and DFR were significantly up-regulated in ML and PL, and the expression of CYP75A in PL and ML was higher than it in $\mathrm{GL}$, but the difference was not significant. Interestingly, the genes associated with stable anthocyanin synthesis were differentially expressed in the GL, ML, and PL, and the expression of the UFGT gene in the GL was much higher than that in the PL and ML. However, qRT-PCR results showed that the UFGT gene behaved extremely differently between the GL and PL. The high expression level of the UFGT gene in PL was consistent with the formation of stable anthocyanins, suggesting that it should be involved in the biosynthesis of anthocyanins[35]. Unlike structural genes, the expression level of candidate regulatory genes (MYB, BHLH, and WD40) was also higher in PL except for BHLH2379, BHLH2808 and WD3208(Fig. 4B). Three MYB genes (MYB1131, MYB1811, and MYB3057) and WD40 proteins (WD2173 and WD3291)were up-regulated in PL, suggesting that MYB and WD factors might be involved in the pigmentation of PL.

\section{Integrative metabolomic and transcriptomic analysis}

As some differentially expressed genes were located in multiple branches, the intensity of metabolite accumulation could not be judged. Therefore, a joint analysis of transcriptome and metabolome was needed to analyze large-scale secondary metabolites and pathway regulation. We focused on the flavonoid biosynthesis pathway and the anthocyanin biosynthesis pathway (Fig. 5).

There were 9 genes involved in anthocyanin biosynthesis, including ANR, CYP75A, DFR, and UFGT. Besides, 11 different metabolites also participated in this process. Based on the differentially expressed genes and metabolites, the metabolic pathway of anthocyanins in L. chinense. var. rubrum was summarized and analyzed. Interestingly, all differentially accumulated anthocyanins, such as cyanidin 3O-glucoside, cyanidin 0-syringic acid, cyanidin 3,5-0-diglucoside, pelargonidin, petunidin 3-0-glucoside, and peonidin 3-sophoroside-5-glucoside, increased in $\mathrm{ML}$ and $\mathrm{PL}$, indicating the presence of anthocyanin branching in ML and PL. Surprisingly, UFGT is directly involved in the stable biosynthesis of anthocyanin synthesis in PL, which is consistent with the accumulation of anthocyanins. In summary, our study showed that DFR, ANR, and UFGT genes played an important role in promoting the accumulation of anthocyanins, including the rise of cyanidin 3-0-glucoside, cyanidin 0-syringic acid, cyanidin 3,5-0diglucoside, pelargonidin, petunidin 3-0-glucoside, and peonidin 3-sophoroside-5-glucoside which brought 
the leaves of $L$. chinense. var. rubrum different colors. Typically, TFs activate genes in secondary metabolic pathways to coordinate their expression[36]. While bHLH was not significantly expressed, MYB (gene1131,3057) and WD40(gene2173,3219) were highly upregulated in PL (Fig. 4B). This is consistent with the trend of the late synergistic genes DFR, ANR, and UFGT. The results had shown that they formed a ternary complex with bHLH and WD40 transcription factors to up-regulate the expression of the DFR, ANR, and UFGT genes, which ultimately formed anthocyanins and greatly improved the level of anthocyanins[37, 38]. In summary, our study showed that DFR, ANR, and UFGT genes played an important role in promoting the accumulation of anthocyanins, including the rise of cyanidien 3-0glucoside, cyanidin 0-syringic acid, cyanidin 3,5-0-diglucoside, pelargonin, petunidin 3-0-glucoside, and peonidin 3-sophoroside-5-glucoside which caused the L. chinense. var. rubrum leaves to have different colors.

\section{Discussion}

L. chinense. var. rubrum, which is a unique ornamental plant in Hunan Province,has a beautiful habit, dazzling flowers, and attractive leaves. Our findings from three different kinds of leaf colors in the same genetic background should help to explain and reveal the molecular mechanisms of color formation.

The color formation of leaves was due to the interaction of pigments such as chlorophyll, anthocyanin, and carotenoid, and the ratio of anthocyanin to chlorophyll content would affect the color presentation of plants[39]. From our findings, the content of the photosynthetic pigment in GL was higher than those in $\mathrm{ML}$ and $\mathrm{PL}$, while the anthocyanin content in $\mathrm{ML}$ and $\mathrm{PL}$ was significantly higher than it in $\mathrm{GL}$. The content of anthocyanins and flavonoids affects the color development of plants[40].

In this study, 241 DEGs and 207 differential metabolites were identified. Nine differential genes, their related metabolites, and their metabolic networks were obtained by analyzing the differential metabolites of differential genes. Thus, changes in downstream metabolites were identified. Our results also indicated that the presence of downstream differential genes of different colors in the leaves was the main initial factor at the transcriptional level. At the metabolic level, metabolic pathways, secondary metabolic biosynthesis, and phenylalanine biosynthesis might also participate in the presentation of leaf color (Additional file 2).

Through analysis of differential metabolites among different phenotypes, anthocyanin was the main metabolite that caused $L$. chinense. var. rubrum leaves show different colors. Combined metabolomics and transcriptome analysis revealed genes involved in anthocyanin synthesis, thus explaining the phenomenon of different colors in L. chinense. var. rubrum leaves. Previous studies on Rose[41], Orchid[42], Chrysanthemum[43], Actinidia arguta[44], Capsicum annuum[45], and Ginkgo biloba[46], etc. had found useful information to explain the formation of different colors of plants.

In this study, DFR, ANR, and UFGT genes were proved to be crucial for the formation of different kinds of anthocyanins and eventually produced different colors. We found that except for the UFGT gene, both DFR and ANR involved in the anthocyanin pathway were significantly up-regulated. In the color 
presentation of $L$. chinense. var. rubrum, the upregulation of DFR and ANR genes was the reason for the accumulation of dyes[47-49]. However, the downregulation of UFGT was in contrast to the qRT-PCR results, which might be related to the regulation of the activity of structural genes by transcription factor genes such as MYBs, BHLH transcription factors, and WD 40 protein[50]. MYB played an important role in regulating early genes[31], besides, MBW complexes were influenced by the late genes for pigment production[51]. In Petunia and other plant species, the temporal, horizontal, and spatial distribution of anthocyanin pigmentation was determined by the MBW complex. PpMYB formed the MBW complex and especially activated UFGT to regulate anthocyanin biosynthesis[52, 53]. Arabidopsis WDrepeat/Mybs/bHLH complex predominantly regulates the expression of the 'late' anthocyanin biosynthetic genes including DFR, LDOX, and UF3GT[31].In this study, two MYB (MYB1131 and MYB3057) and two WD40 (WD2173 and WD3291) were strikingly upregulated in PL (Table3; Table S5).

The up-regulated MYB and WD40 transcription factors will activate the expression of structural genes involved in the anthocyanin biosynthetic pathway, leading to the increase of anthocyanin concentrations. Present results might indicate that the structural genes and transcription factors that regulate the production of secondary metabolites are significantly different in the samples. However, it is necessary to further study how transcription factors and structural genes lead to changes in leaf color. This will decipher the systematic regulation mechanism of $L$. chinense. var. rubrum leaf coloring and be beneficial to the transgenic engineering of other plants.

\section{Conclusion}

In this study, the transcriptome and metabolome of three colored L. chinense. var. rubrum leaves were sequenced and analyzed for the first time. We observed substantial increases in levels of cyanidin 3-0glucoside, cyanidin 0-syringic acid, Cyanidin 3,5-0-diglucoside, pelargonidin, petunidin 3-0-glucoside, peonidin 3-sophoroside-5-glucoside. The high content of anthocyanins in ML and PL was associated with an upregulation of genes involved in the latter steps of anthocyanin synthesis (DFR, ANR, and UFGT). Several transcription factors such as MYB and WD40 were predicted to be the main regulators in the anthocyanin biosynthetic structural genes.

In summary, our results proved that the expression of several regulatory and structural genes could promote the anthocyanin biosynthesis, and provided the sequence information for their leaf color change and metabolite base.

\section{Materials And Methods}

\section{Plant material}

In this study, L. chinense var. rubrum plants were provided by Hunan Mid-subtropical Quality Plant Breeding and Utilization Engineering Technology Research Center, Hunan Agricultural University. We selected accession number Xiang S-SV-LC-032-2012-1 as mosaic leaves plant, accession number Xiang S-SV-LC-032-2012-2 as purple leaves plant, and accession number Xiang S-SV-LC-032-2012-3 as green 
leaves plants. We named them ML, PL and GL, respectively, in this study. Plants were grown in the Garden Flower Base of Hunan Agricultural University, Changsha, Hunan Province, China. ( $\left.28^{\circ} 12^{\prime} \mathrm{N}, 112^{\circ} 59^{\prime} \mathrm{E}\right)$. These samples were immediately frozen in liquid nitrogen and stored at $-80^{\circ} \mathrm{C}$ before metabolomic and transcriptomic analyses. The research materials were as shown in Fig. 1a. The GL was the control group, and the $M L$ and the PL were the experimental groups. Besides, three apical middle and upper leaves per color selection were used, half samples were sent to Wuhan Netware for metabolome determination and the metabolites were qualitatively and quantitatively analyzed based on UPLC-Q-trap/MS detection platform and extensive targeted metabolome detection technology. And the other half samples for transcriptome sequencing were stored in liquid nitrogen and sent to Beijing Novogene for transcriptome sequencing. Three biological replicates of $G L, M L$, and $P L$ were recorded as samples GL1-3, ML1-3, and PL1-3.

\section{Determination of photosynthetic and anthocyanin content}

Chlorophyll content and carotenoid content in the leaves of $\mathrm{GL}, \mathrm{ML}$, and $\mathrm{PL}$ of $L$. chinense. var. rubrum measured directly by extraction method. After the veins were removed, $0.1 \mathrm{~g}$ of each kind of leaves was weighed, then $15 \mathrm{ml}$ of $95 \%$ alcohol was added, and every leaf tissue was put at $4{ }^{\circ} \mathrm{C}$ for whitening in the dark, finally, the supernatant was taken. The absorbance values at $470 \mathrm{~nm}, 649 \mathrm{~nm}$, and $665 \mathrm{~nm}$ were measured with an ultraviolet spectrophotometer (AOE TSD-599), and each variety was repeated six times. Anthocyanin was detected by the $\mathrm{pH}$ differential method for cyanidin-3-glucoside content in plants. The revised method proposed by Zhang et al. was adopted[54], and the calculation formula is as follows:

$\mathrm{Ca}=13.95 \mathrm{~A}_{665}-6.88_{649} ; \mathrm{Cb}=24.96 \mathrm{~A}_{649}-7.32_{\mathrm{A} 665} ; \mathrm{CC}=\left(1000_{\mathrm{A} 470}-2.05_{\mathrm{Ca}}-114.8_{\mathrm{Cb}}\right) / 245$

$T A=A * M W * 5 * 100 * V / e[55]$.

\section{Extraction and NMR of Anthocyanin}

$0.1 \mathrm{~g}$ of freeze-dried leaf sample apriority was weighed and transferred into centrifuge tubes next, these samples were pulverized with a grinder at $30 \mathrm{~Hz}$ for $1.5 \mathrm{~min}$. Then, the powdered sample was extracted overnight at $4^{\circ} \mathrm{Cby}$ adding $1.0 \mathrm{ml}(70 \%$ of aqueous methanol). Samples were centrifuged at $10000 \mathrm{~g}$ for $10 \mathrm{~min}$, then absorbed by the supernatant filter with 0.22 microporous filter membrane, and finally stored in the sample bottle for LC-MS/MS analysis. Chromatographic separation was performed on a water ACQUITY UPLC HSS T3 C18(1.8 m,2.1 mm*100 mm) using solvent A (water,0.04\% acetic acid): solvent B (acetonitrile, $0.04 \%$ acetic acid). The solvent system consisted of a water: acetonitrile gradient programming 100:0V/V for 0 minutes,5:95V/V for 11.0 minutes,5:95V/V for 12.0 minutes,95:5V/V for 12.1 minutes, $95: 5 \mathrm{~V} / \mathrm{V}$ for 15.0 minutes. The flow rate was $0.40 \mathrm{ml} . \mathrm{min}^{-1}$, the Column temperature was $40^{\circ} \mathrm{Cand}$ the injection volume was $5 \mu \mathrm{L}$. The effluent was alternately connected to electrospray-triple quadrupole rod-linear ion trap-mass spectrometry. The ESI source temperature was $550^{\circ} \mathrm{C}$; lon spray voltage 5500 volts, and the CUR was set to 55,60 and 25.0 psi respectively.

The structure analysis of metabolites was from the Mass Bank (http://www.massbank.jp/), KNAPSACK (HTTP://kanaya,naist,jp/KNAPSACK/), HMDB (HTTP//www.hmdb.cal) 
[56],MOTODB(http://www.ab.wur.nl/moto) and METLIN (http://metlin.scripps.edu/index.php[57]. The identified metabolites based on the KEGG pathway database (http://www.genome,jp/kegg/pathway.html) were to identify related metabolic pathways[32]. Qualitative analysis of spectral and secondary spectral data was carried out through a self-built database MWDB and a public database of metabolite information. Metabolite quantification was based on MRM using triple quadrupole mass spectrometry.

\section{RNA extraction, library construction and RNA-Seq}

Total RNA was extracted from approximately $2 \mathrm{~g}$ of $L$. chinense. var. rubrum leaves by using Gene Star (Beijing, China). Library preparation and transcriptome sequencing were performed by Novogene Bioinformatics Technology (Beijing, China). The libraries were sequenced by using an Illumina HiSeq platform and the raw reads filtering the adaptor sequence, lower quality (the quality value is less than 20), and $\mathrm{N}$-containing reads followed by de novo assembly of the transcriptome using Trinity. The unigene for functional annotation, which was obtained by alignment of the unigene with the Go, KOG, KEGG, Nt, Nr, and Pfam database to annotate their potential metabolic pathways.

Expression levels of genes related to leaf color formation of $L$. chinense. var. rubrum was explored with the RSEM software package[58, 59]. To evaluate the expression abundance of the unigene by the FPKM method[60]. Differential expression analyses of the leaf colors were performed by the DESeq $R$ package(1.10.1).DEGs were those who had $\mid \log _{2}$ (fold change) $\mid \geq 2$,FDR value $<0.01$.Go and $K E G G$ pathway enrichment analysis of the DEGs was done by the GOseq R package-based hypergeometric distribution[61], which can adjust gene length bias in DEGs.

\section{Quantitative real-time PCR (qRT-PCR) analysis}

To further confirm the reliability of RNA-seq data in our differential expression analysis, 10 structural genes in the flavonoid metabolic pathway were selected to analyze the relative expression levels of $L$. chinense. var. rubrum in three-color leaves through qRT-PCR. Primer was designed with Primer Premier 5.0 software, and the details were shown in Additional file 9. With the $\beta$-actin gene as an internal reference gene, three technical replicates were set for each biological sample, and the expression level of each gene in the list was calculated by the $2^{-} \Delta \Delta \mathrm{Ct}$ method and expressed as the average standard deviation.

\section{Integrative metabolomic and transcriptomic analysis}

Transcriptomic and metabolomic data for $L$. chinense. var. rubrum leaves with clear differences were used for analysis. Correlation analysis was carried out according to the metabolite content of different colors of leaves in metabolic data and the differential gene expression in transcriptome data.

\section{Abbreviations}

L. chinense var. rubrum: Loropetalum chinense var. rubrum; GL: green leaves; ML: mixed leaves; PL: pink leaves; Ca: Chlorophyll a; Cb: Chlorophyll b; Cc: Carotenoid concentration; TA: Total anthocyanin; PAL: 
phenylalanine ammonia-lyase; C4H: cinnamate 4-hydroxylase; 4CL:4-coumaroyl: CoA-ligase; CHS: chalcone synthase; $\mathrm{CHI}$ : chalcone isomerase; $\mathrm{F3H}$;flavanone 3-hydroxylase; ANR: anthocyanidin reductase; CYP75A:flavonoid 3',5'-hydroxylase; DFR: dihydro-flavonol 4-reductase; UFGT:3-3-0glycosyltransferase; MYB: MYB transcription factor; bHLH: basic helix-loop-helix transcription factor; WD40هWD40 proteins; FPKM: fragment per kilobase per million reads; Log2FC; Log2 fold change; DEGs: differentially expressed genes; qRT-PCR: quantitative real-time PCR; Go: Gene ontology; KOG: eukaryotic Ortholog Groups; KEGG: Kyoto Encyclopedia of Gene and Genomes; Nt: NCBI nucleotide sequences; Nr: NCBI nonredundant protein; Pfam: Protein family; MRM: multiple reaction monitoring; CUR: curtain gas; MWDB: met ware database;

\section{Declarations}

\section{Ethics approval and consent to participate}

Not applicable.

\section{Consent for publication}

Not applicable.

\section{Availability of data and material}

All data generated/analyzed during this study are included in this article and its supplementary files.

\section{Competing interests}

The authors declare that the research was conducted in the absence of any commercial or financial relationships that could be construed as a potential conflict of interest.

\section{Funding}

This study was conducted by Hunan Provincial Science and Technology Plan (Grant No.2018TP2007), Hunan Province Key Research and Development Program of Science and Technology Department (Grant No.2016NK2100), Hunan Agriculture Establishment, Research and Cultivation of Universities' Shuang Yi Current'(Grant No. SYL201802026 and SYL2019012), Hunan province education department project(Grant No.18B124) and Hu Nan agricultural university the third batch of major scientific research projects and a New team cultivation project (Grant No.201909). The funding bodies played no role in the design of the study and collection, analysis, and interpretation of data and in writing the manuscript.

\section{Authors' contributions}

X.Z., L.Z., and D.M.Z collected the sample conceived and designed the study. X.Z., L.Z., D.D.S., and W.D.L the genome annotation and functional genomic analysis. X.F.W., Q.R.C., W.Q.C., L.X., and F.X.C performed 
the data analysis of the metabolome. X.Z., X.Y.Y., Y.L.L., and D.L.Z wrote the manuscript. All the authors reviewed and approved the manuscript.

Acknowledgments

Not applicable.

\section{References}

1. Zhang W, Zhang C, Li M, Du W, Shao H, Yang S, Feng Y, Chen H: Phytochemical and chemotaxonomic study on Loropetalum chinense (R. Br.) Oliv. Biochemical Systematics and Ecology 2018, 81:80-82.

2. Zhou G, Yu H, Lu C, Zhou Z: Primary Study on Bacteriostatic Active Compound from Leaves of Loropetalum chinense var. rubrum Yieh. Food Sciense 2007(06):74-77.

3. Tang h, Zheng Q, Liang T, Zhong T, Huang I, Ge G, Sun B: Analysis of Volatile Oils in Leaves of Loropetalum chinense and L.chinense var.rubrum Using Simultanuous Distillation and Solvent Extraction (SDE) and GC-MS Journal of Anhui Agri 2011, 39(26):15985-15987+15990.

4. Tanaka Y, Brugliera F, Kalc G, Senior M, Dyson B, Nakamura N, Katsumoto Y, Chandler S: Flower Color Modification by Engineering of the Flavonoid Biosynthetic Pathway: Practical Perspectives. Bioscience Biotechnology and Biochemistry 2010, 74(9):1760-1769.

5. Zhu M, Zheng X, Shu Q, Li H, Zhong P, Zhang H, Xu Y, Wang L, Wang L: Relationship between the Composition of Flavonoids and Flower Colors Variation in Tropical Water Lily (Nymphaea) Cultivars. Plos One 2012, 7(4).

6. Zhao D, Tao J, Han C, Ge J: Flower color diversity revealed by differential expression of flavonoid biosynthetic genes and flavonoid accumulation in herbaceous peony (Paeonia lactiflora Pall.). Molecular Biology Reports 2012, 39(12):11263-11275.

7. Liu X, Liu C, Lu X, Tong Z, Tao C, Yuan X: Comprehensive Transcriptome Analyses of Carbohydrate Metabolism and Flavonoid Biosynthesis in Blueberry (Vaccinium corymbosum) during Fruit Maturation. International Journal of Agriculture and Biology 2020, 24(1):101-111.

8. Lin Q, Zhong Q, Zhang Z: Comparative transcriptome analysis of genes involved in anthocyanin biosynthesis in the pink-white and red fruits of Chinese bayberry (Morella rubra). Scientia Horticulturae 2019, 250:278-286.

9. Deng C, Li S, Feng C, Hong Y, Huang H, Wang J, Wang L, Dai S: Metabolite and gene expression analysis reveal the molecular mechanism for petal colour variation in six Centaurea cyanus cultivars. Plant Physiology and Biochemistry 2019, 142:22-33.

10. Li L, Zhai Y, Luo X, Zhang Y, Shi Q: Comparative transcriptome analyses reveal genes related to pigmentation in the petals of red and white Primula vulgaris cultivars. Physiology and Molecular Biology of Plants 2019, 25(4):1029-1041. 
11. Zhang C, Li X, Zhan Z, Cao L, Zeng A, Chang G, Liang Y: Transcriptome Sequencing and Metabolism Analysis Reveals the role of Cyanidin Metabolism in Dark-red Onion (Allium cepa L.) Bulbs. Scientific Reports 2018, 8.

12. Asem ID, Imotomba RK, Mazumder PB, Laishram JM: Anthocyanin content in the black scented rice (Chakhao): its impact on human health and plant defense. Symbiosis 2015, 66(1):47-54.

13. Sudheeran PK, Feygenberg O, Maurer D, Alkan N: Improved Cold Tolerance of Mango Fruit with Enhanced Anthocyanin and Flavonoid Contents. Molecules 2018, 23(7).

14. Nakabayashi R, Yonekura-Sakakibara K, Urano K, Suzuki M, Yamada Y, Nishizawa T, Matsuda F, Kojima M, Sakakibara H, Shinozaki $\mathrm{K}$ et al: Enhancement of oxidative and drought tolerance in Arabidopsis by overaccumulation of antioxidant flavonoids. Plant Journal 2014, 77(3):367-379.

15. Mattioli R, Francioso A, Mosca L, Silva P: Anthocyanins: A Comprehensive Review of Their Chemical Properties and Health Effects on Cardiovascular and Neurodegenerative Diseases. Molecules 2020, 25(17).

16. Xu D, Pan Y, Chen J: Chemical Constituents, Pharmacologic Properties, and Clinical Applications of Bletilla striata. Frontiers in Pharmacology 2019, 10.

17. Sulc M, Kotikova Z, Paznocht L, Pivec V, Hamouz K, Lachman J: Changes in anthocyanidin levels during the maturation of color-fleshed potato (Solanum tuberosum L.) tubers. Food Chemistry 2017, 237:981-988.

18. Luo Q, Liu R, Zeng L, Wu Y, Jiang Y, Yang Q, Nie Q: Isolation and molecular characterization of NtMYB4a, a putative transcription activation factor involved in anthocyanin synthesis in tobacco. Gene 2020, 760.

19. Winkel-Shirley B: Flavonoid biosynthesis. A colorful model for genetics, biochemistry, cell biology, and biotechnology. Plant physiology 2001, 126(2):485-493.

20. Meng X, Li G, Gu L, Sun Y, Li Z, Liu J, Wu X, Dong T, Zhu M: Comparative Metabolomic and Transcriptome Analysis Reveal Distinct Flavonoid Biosynthesis Regulation Between Petals of White and Purple Phalaenopsis amabilis. Journal of Plant Growth Regulation 2020, 39(2):823-840.

21. Peng Y, Thrimawithana AH, Cooney JM, Jensen DJ, Espley RV, Allan AC: The proanthocyanin-related transcription factors MYBC1 and WRKY44 regulate branch points in the kiwifruit anthocyanin pathway. Scientific Reports 2020, 10(1).

22. Tanaka Y, Sasaki N, Ohmiya A: Biosynthesis of plant pigments: anthocyanins, betalains and carotenoids. Plant Journal 2008, 54(4):733-749.

23. Zhao ZC, Hu GB, Hu FC, Wang HC, Yang ZY, Lai B: The UDP glucose: flavonoid-3-Oglucosyltransferase (UFGT) gene regulates anthocyanin biosynthesis in litchi (Litchi chinesis Sonn.) during fruit coloration. Molecular Biology Reports 2012, 39(6):6409-6415.

24. Liu X, Huang Y, Qiu Z, Gong H: Comparative transcriptome analysis of differentially expressed genes between the fruit peel and flesh of the purple tomato cultivar 'Indigo Rose'. Plant Signaling \& Behavior 2020, 15(6). 
25. Wang P, Chen S, Gu M, Chen X, Chen X, Yang J, Zhao F, Ye N: Exploration of the Effects of Different Blue LED Light Intensities on Flavonoid and Lipid Metabolism in Tea Plants via Transcriptomics and Metabolomics. International Journal of Molecular Sciences 2020, 21(13).

26. Yang K, Li Y, Wang S, Xu X, Sun H, Zhao H, Li X, Gao Z: Genome-wide identification and expression analysis of the MYB transcription factor in moso bamboo (Phyllostachys edulis). PeerJ 2019, 6.

27. Wang L, Huang Q, Zhang L, Wang Q, Liang L, Liao B: Genome-Wide Characterization and Comparative Analysis of MYB Transcription Factors in Ganoderma Species. G3-Genes Genomes Genetics 2020, 10(8):2653-2660.

28. Roy S: Function of MYB domain transcription factors in abiotic stress and epigenetic control of stress response in plant genome. Plant Signaling \& Behavior 2016, 11(1).

29. An X-H, Tian Y, Chen K-Q, Wang X-F, Hao Y-J: The apple WD40 protein MdTTG1 interacts with bHLH but not MYB proteins to regulate anthocyanin accumulation. Journal of Plant Physiology 2012, 169(7):710-717.

30. Albert NW: Subspecialization of R2R3-MYB Repressors for Anthocyanin and Proanthocyanidin Regulation in Forage Legumes. Frontiers in Plant Science 2015, 6.

31. Gonzalez A, Zhao M, Leavitt JM, Lloyd AM: Regulation of the anthocyanin biosynthetic pathway by the TTG1/bHLH/Myb transcriptional complex in Arabidopsis seedlings. Plant Journal 2008, 53(5):814-827.

32. Chen Y, Zhang R, Song Y, He J, Sun J, Bai J, An Z, Dong L, Zhan Q, Abliz Z: RRLC-MS/MS-based metabonomics combined with in-depth analysis of metabolic correlation network: finding potential biomarkers for breast cancer. Analyst 2009, 134(10):2003-2011.

33. Kanehisa M: Enzyme Annotation and Metabolic Reconstruction Using KEGG. Methods in molecular biology (Clifton, NJ) 2017, 1611:135-145.

34. Jiao F, Zhao L, Wu X, Song Z, Li Y: Metabolome and transcriptome analyses of the molecular mechanisms of flower color mutation in tobacco. Bmc Genomics 2020, 21(1).

35. Ford CM, Boss PK, Hoj PB: Cloning and characterization of Vitis vinifera UDP-glucose:flavonoid 3-0glucosyltransferase, a homologue of the enzyme encoded by the maize Bronze-1 locus that may primarily serve to glucosylate anthocyanidins in vivo. The Journal of biological chemistry 1998 , 273(15):9224-9233.

36. Cao Y, Li K, Li Y, Zhao X, Wang L: MYB Transcription Factors as Regulators of Secondary Metabolism in Plants. Biology-Basel 2020, 9(3).

37. Kobayashi S, Ishimaru M, Hiraoka K, Honda C: Myb-related genes of the Kyoho grape (Vitis labruscana) regulate anthocyanin biosynthesis. Planta 2002, 215(6):924-933.

38. Kaur R, Kapoor N, Aslam L, Mahajan R: Molecular characterization of PgUFGT gene and R2R3PgMYB transcription factor involved in flavonoid biosynthesis in four tissues of wild pomegranate (Punica granatum L.). Journal of Genetics 2019, 98(4).

39. Song B, Xu H, Chen L, Fan X, Jing Z, Chen S, Xu Z: Study of the Relationship between Leaf Color Formation and Anthocyanin Metabolism among Different Purple Pakchoi Lines. Molecules 2020, 
25(20).

40. Wang Z, Cui Y, Vainstein A, Chen S, Ma H: Regulation of Fig (Ficus carica L.) Fruit Color: Metabolomic and Transcriptomic Analyses of the Flavonoid Biosynthetic Pathway. Frontiers in Plant Science 2017, 8.

41. Huang P, Lin F, Li B, Zheng Y: Hybrid-Transcriptome Sequencing and Associated Metabolite Analysis Reveal Putative Genes Involved in Flower Color Difference in Rose Mutants. Plants-Basel 2019, 8(8).

42. Zhang Y, Zhou T, Dai Z, Dai X, Li W, Cao M, Li C, Tsai W-C, Wu X, Zhai J et al: Comparative Transcriptomics Provides Insight into Floral Color Polymorphism in a Pleione limprichtii Orchid Population. International Journal of Molecular Sciences 2020, 21(1).

43. Kim D-H, Park S, Park B-R, Lee J-Y, Lim S-H: Flavonoid Metabolic Engineering for Modification of Flower Color in Chrysanthemum. Korean Journal of Breeding Science 2018, 50(4):351-363.

44. Li Y, Fang J, Qi X, Lin M, Zhong Y, Sun L, Cui W: Combined Analysis of the Fruit Metabolome and Transcriptome Reveals Candidate Genes Involved in Flavonoid Biosynthesis in Actinidia arguta. International Journal of Molecular Sciences 2018, 19(5).

45. Liu Y, Lv J, Liu Z, Wang J, Yang B, Chen W, Ou L, Dai X, Zhang Z, Zou X: Integrative analysis of metabolome and transcriptome reveals the mechanism of color formation in pepper fruit (Capsicum annuum L.). Food Chemistry 2020, 306.

46. Guo J, Wu Y, Wang G, Wang T, Cao F: Integrated analysis of the transcriptome and metabolome in young and mature leaves of Ginkgo biloba L. Industrial Crops and Products 2020, 143.

47. Dai LP, Dong XJ, Ma HH: Molecular mechanism for cadmium-induced anthocyanin accumulation in Azolla imbricata. Chemosphere 2012, 87(4):319-325.

48. Wang H, Fan W, Li H, Yang J, Huang J, Zhang P: Functional Characterization of Dihydroflavonol-4Reductase in Anthocyanin Biosynthesis of Purple Sweet Potato Underlies the Direct Evidence of Anthocyanins Function against Abiotic Stresses. Plos One 2013, 8(11).

49. Trainin T, Harel-Beja R, Bar-Ya'akov I, Ben-Simhon Z, Yahalomi R, Borochov-Neori H, Ophir R, Sherman A, Doron-Faigenboim A, Holland D: Fine Mapping of the "black" Peel Color in Pomegranate (Punica granatum L.) Strongly Suggests That a Mutation in the Anthocyanidin Reductase (ANR) Gene Is Responsible for the Trait. Frontiers in Plant Science 2021, 12.

50. Broun P: Transcriptional control of flavonoid biosynthesis: a complex network of conserved regulators involved in multiple aspects of differentiation in Arabidopsis. Current opinion in plant biology 2005, 8(3):272-279.

51. Borevitz JO, Xia Y, Blount J, Dixon RA, Lamb C: Activation tagging identifies a conserved MYB regulator of phenylpropanoid biosynthesis. The Plant cell 2000, 12(12):2383-2394.

52. Feng $S$, Wang $Y$, Yang $S, X u Y, C h e n X$ : Anthocyanin biosynthesis in pears is regulated by a R2R3MYB transcription factor PyMYB10. Planta 2010, 232(1):245-255.

53. Qian M, Sun Y, Allan AC, Teng Y, Zhang D: The red sport of 'Zaosu' pear and its red-striped pigmentation pattern are associated with demethylation of the PyMYB10 promoter.

Phytochemistry 2014, 107:16-23. 
54. Zhang Y-L, Fang Z-Z, Ye X-F, Pan S-L: Identification of candidate genes involved in anthocyanin accumulation in the peel of jaboticaba (Myrciaria cauliflora) fruits by transcriptomic analysis. Gene 2018, 676:202-213.

55. Romero I, Sanchez-Ballesta MT, Maldonado R, Escribano MI, Merodio C: Anthocyanin, antioxidant

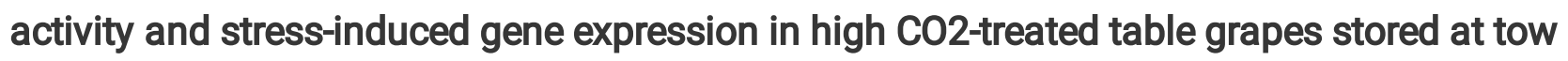
temperature. Journal of Plant Physiology 2008, 165(5):522-530.

56. Wishart DS, Jewison T, Guo AC, Wilson M, Knox C, Liu Y, Djoumbou Y, Mandal R, Aziat F, Dong E et al: HMDB 3.0-The Human Metabolome Database in 2013. Nucleic Acids Research 2013, 41(D1):D801D807.

57. Zhu Z-J, Schultz AW, Wang J, Johnson CH, Yannone SM, Patti GJ, Siuzdak G: Liquid chromatography quadrupole time-of-flight mass spectrometry characterization of metabolites guided by the METLIN database. Nature Protocols 2013, 8(3):451-460.

58. Li B, Dewey CN: RSEM: accurate transcript quantification from RNA-Seq data with or without a reference genome. Bmc Bioinformatics 2011, 12.

59. Anders S, Huber W: Differential expression analysis for sequence count data. Genome Biology 2010, 11(10).

60. Mortazavi A, Williams BA, McCue K, Schaeffer L, Wold B: Mapping and quantifying mammalian transcriptomes by RNA-Seq. Nature Methods 2008, 5(7):621-628.

61. Mao X, Cai T, Olyarchuk JG, Wei L: Automated genome annotation and pathway identification using the KEGG Orthology (KO) as a controlled vocabulary. Bioinformatics (Oxford, England) 2005, 21(19):3787-3793.

\section{Figures}


A
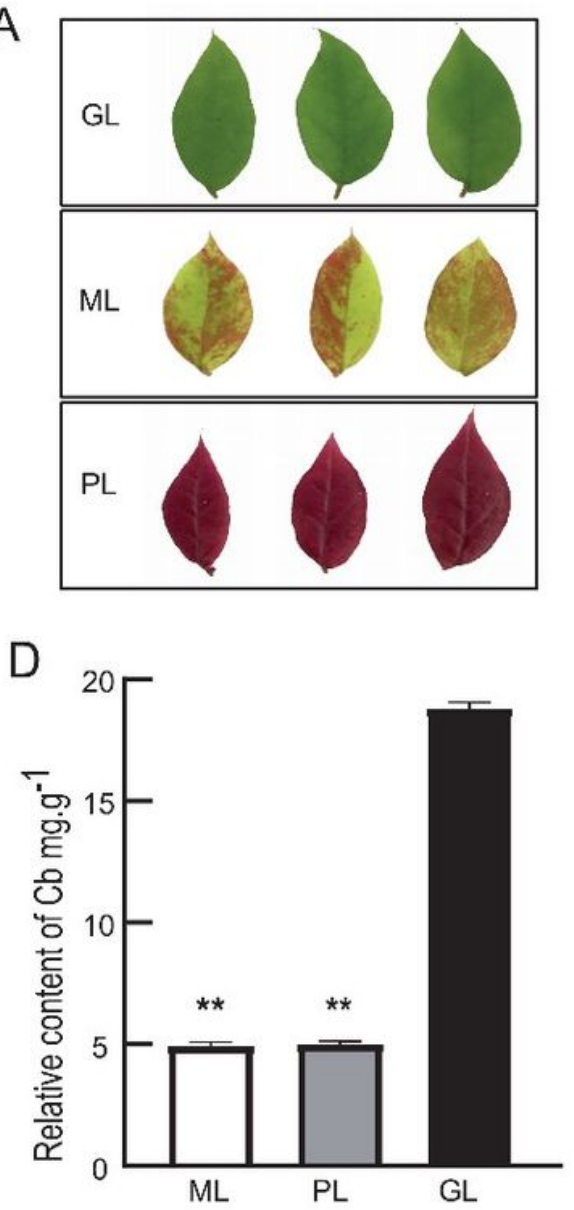
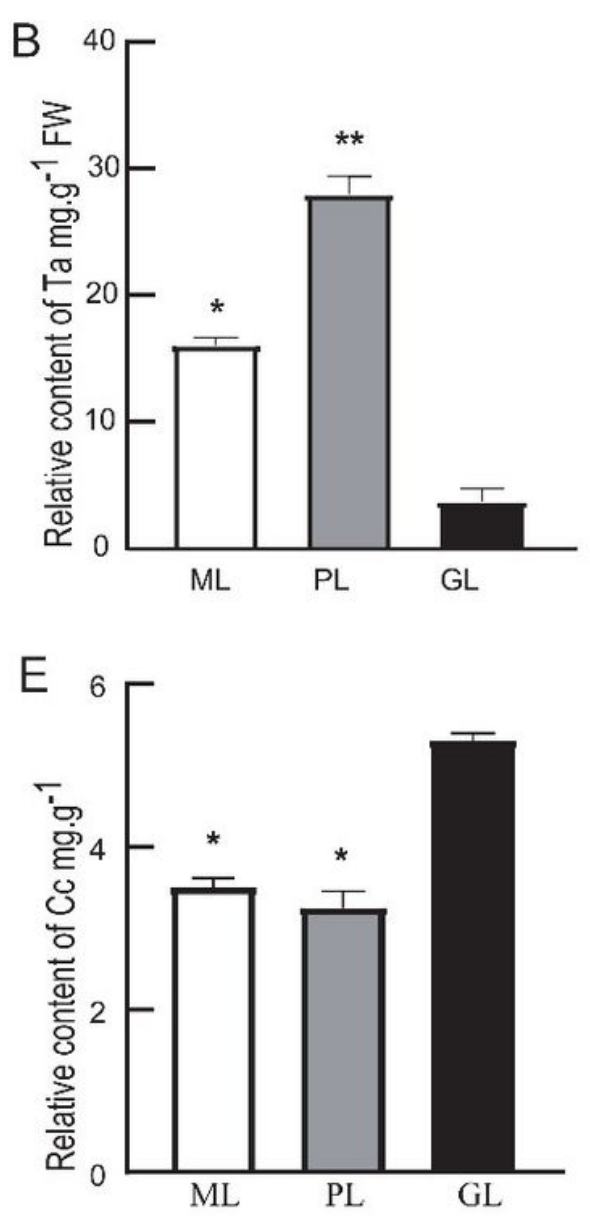
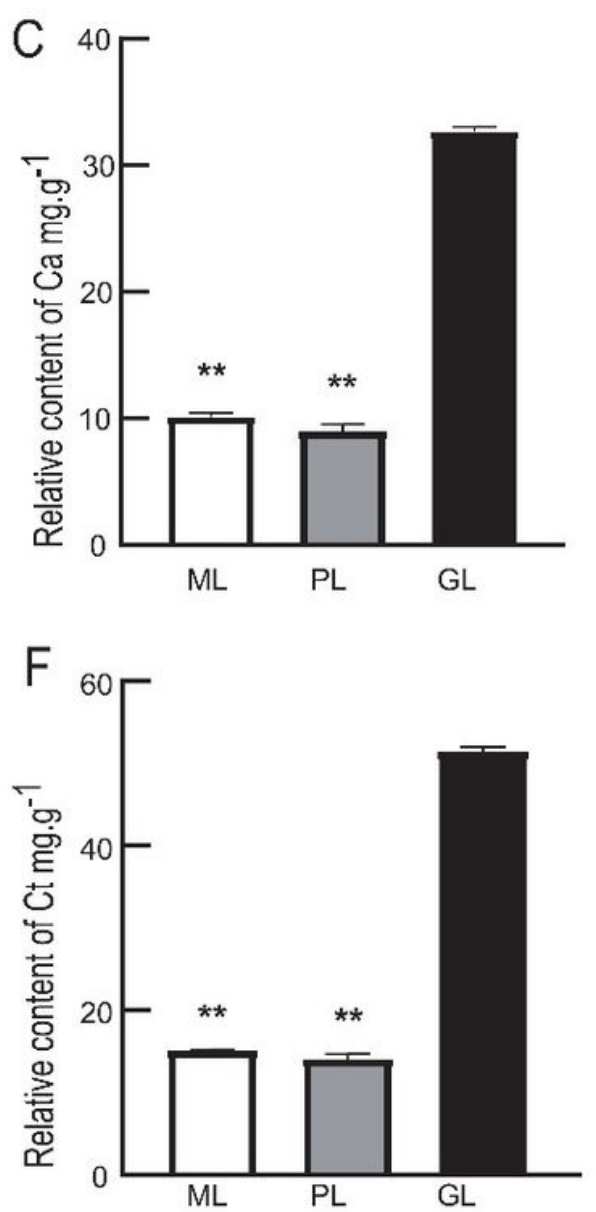

\section{Figure 1}

Determination of photosynthetic and anthocyanin content. A Leaf colors pf the three-leaf colors $G L, M L$, and PL. B Relative content of total anthocyanins. C Relative content of chlorophyll A. D Relative content of chlorophyll B. E Relative contents of Carotenoid concentration. F Relative contents of total chlorophyll. The green leaf was used as the control group for three biological repetitions, and the value was subject to the means \pm standard. Asterisks indicate statistically significant differences (Student's t-test; ${ }^{*} \mathrm{P} \leq 0.05$ and $* * P)$ 
A

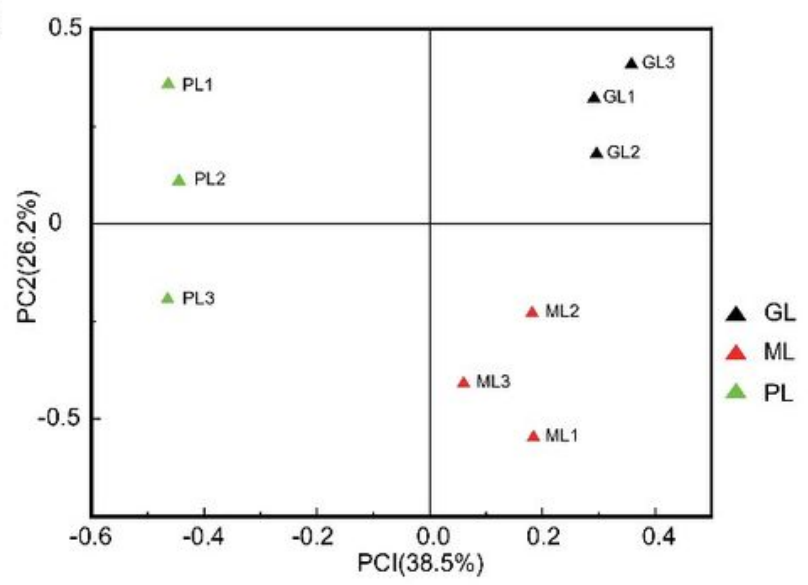

C

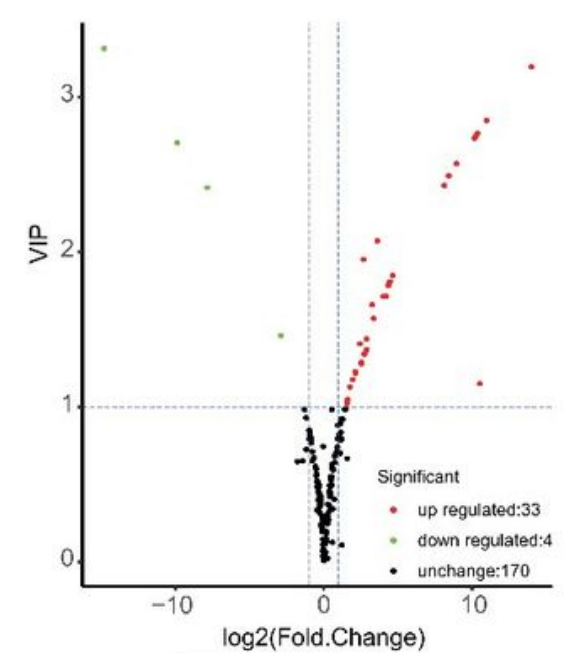

E

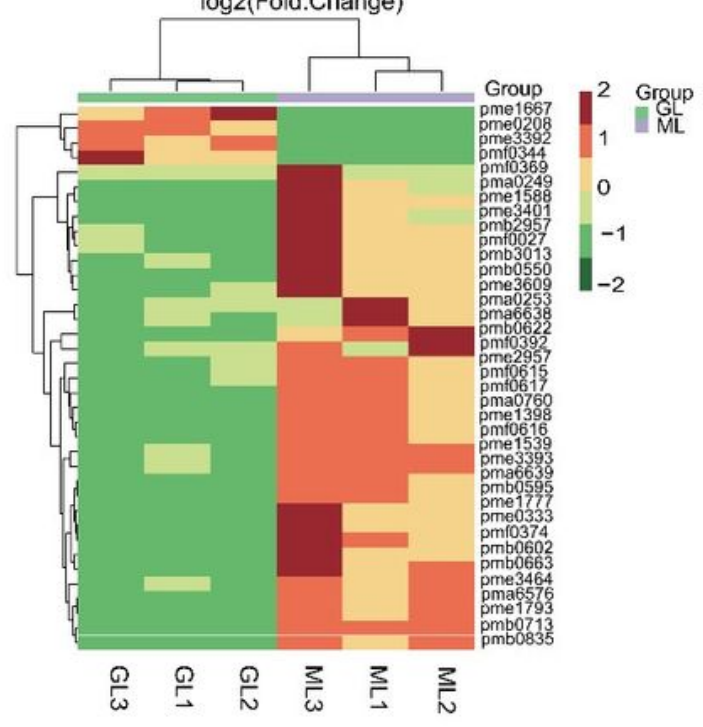

B

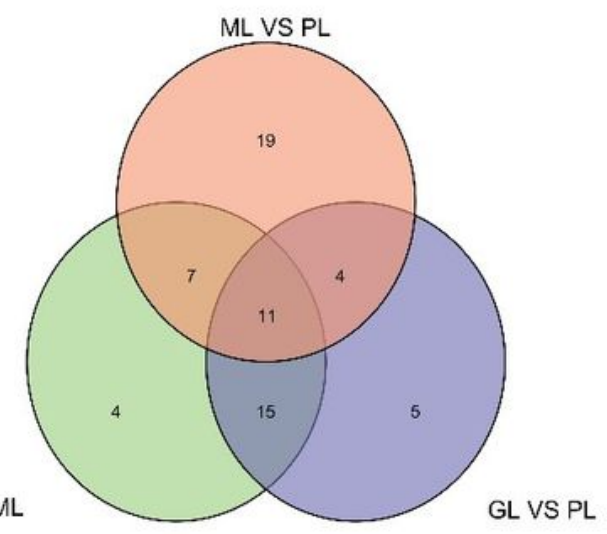

D

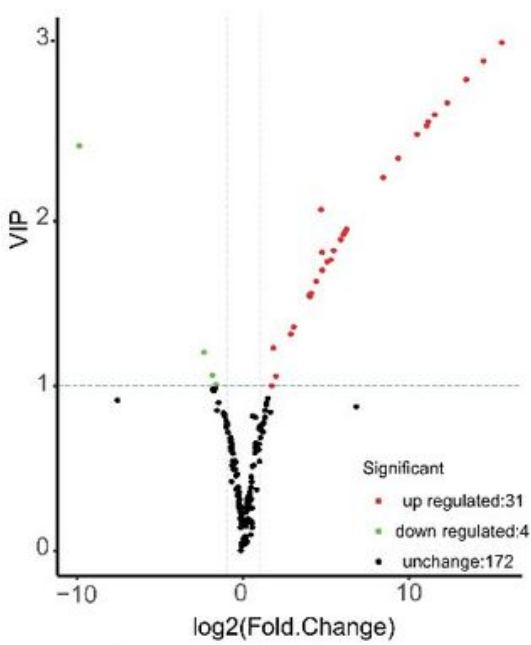

$\mathrm{F}$

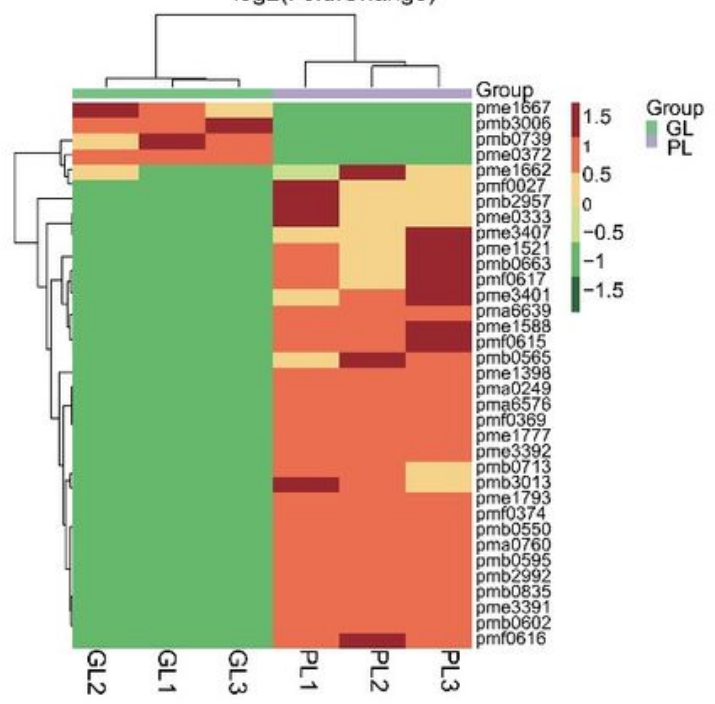

\section{Figure 2}

Differential metabolites from different leaves. A PCA score plot of three materials and numbers of potential markers for each leaf color. B Venn diagram of SCMs. C Volcano plots of SCMs between GL and ML. D Volcano plots of SCMs between GL and PL. E Cluster heat map of SCMs between GL and ML. F Cluster heat map of SCMs between GL and PL 

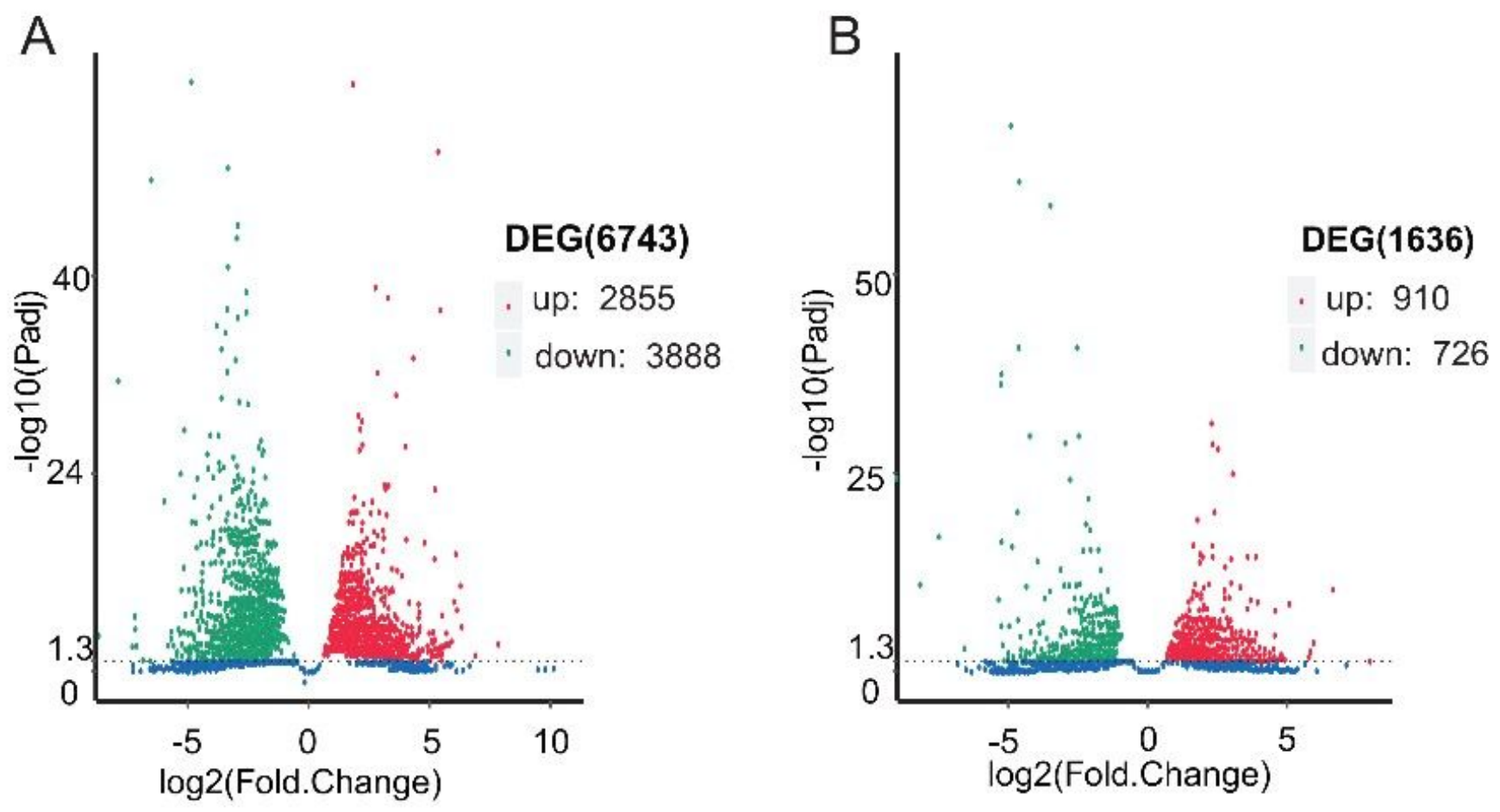

Figure 3

Differential expression genes in different colors A, B. The volcano plot shows the differential expression gene between $\mathrm{ML}, \mathrm{PL}$, and $\mathrm{GL}$

A
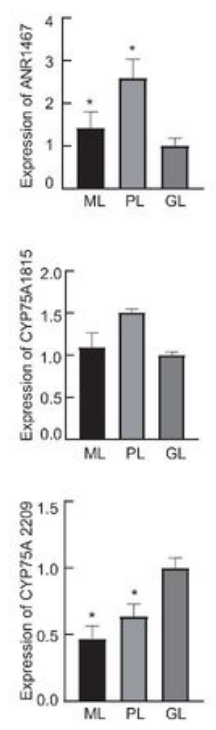
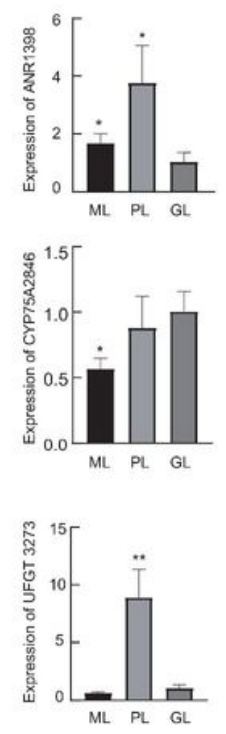
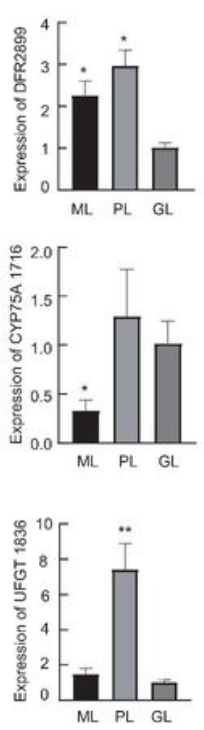

B
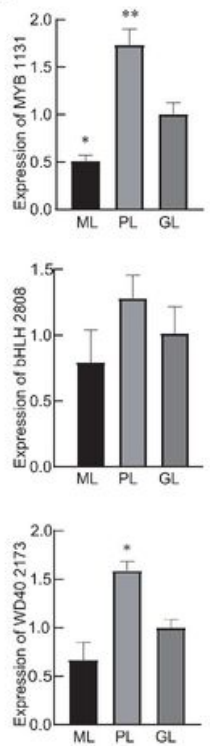
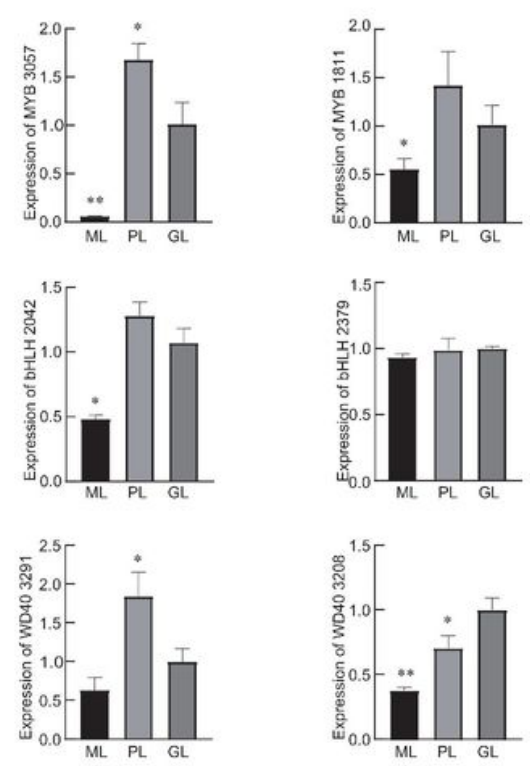

Figure 4

Transcriptional levels of flavonoid biosynthetic genes in the $L$. chinense. var. rubrum detected by qRT-PCR analysis. The $\beta$-actin gene was used as an internal control. A ANR; UFGT, DFR, and CYP75A. B MYB, 
bHLH, WD40. The control for relative expression GL was assigned the arbitrary value of 1.0. Values presented are the means \pm standard errors of three analyses for two biological replications. Asterisks indicate statistically significant differences (Student's t-test; *P $\leq 0.05$ and $* * P$ )

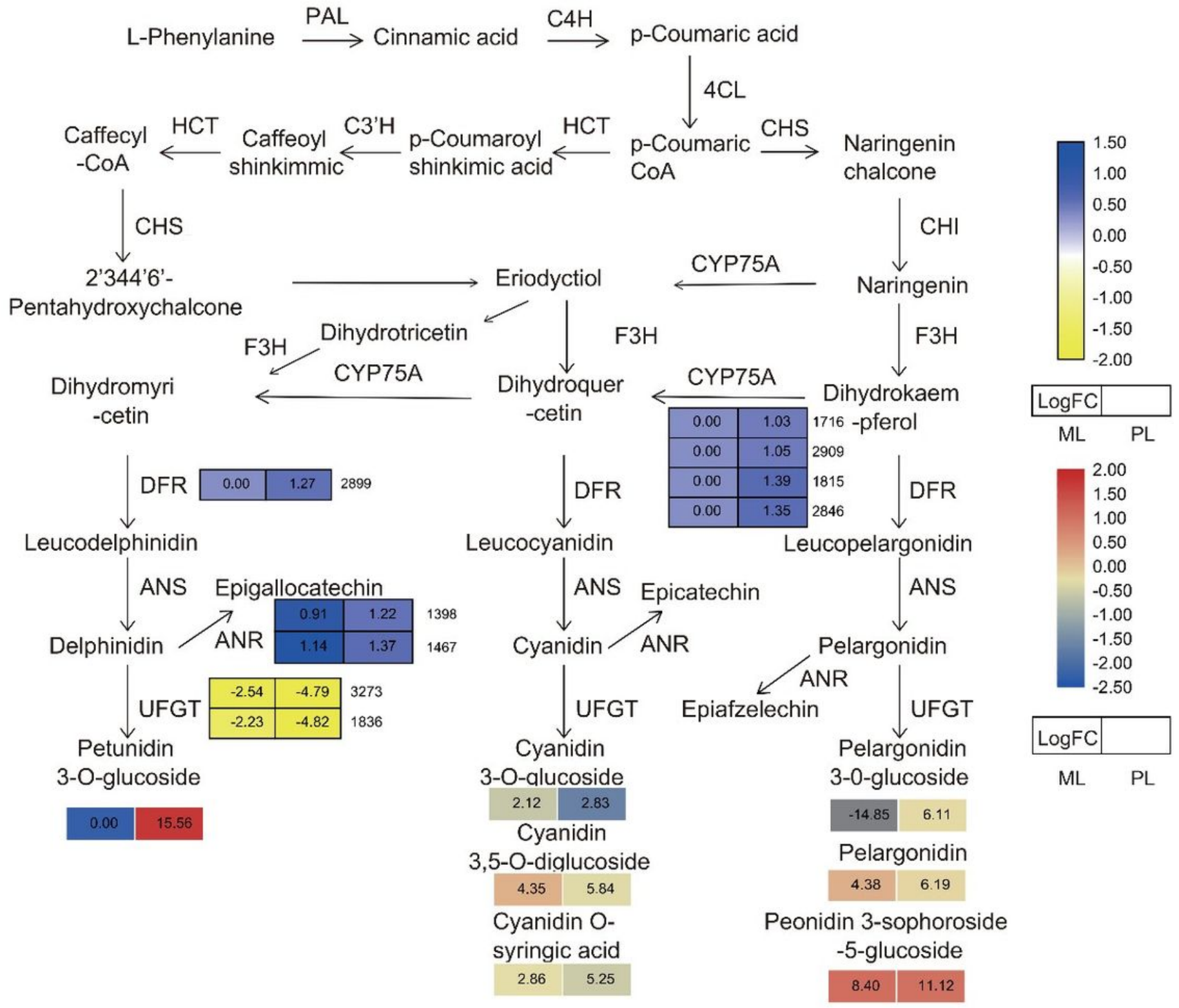

\section{Figure 5}

Regulatory network of flavonoid biosynthesis in L. chinense. var. rubrum. The left block below each gene/metabolite is the Log2FC of this between GL vs ML. The right block below each gene/metabolite is the Log2FC of this between GL vs PL

\section{Supplementary Files}

This is a list of supplementary files associated with this preprint. Click to download. 
- Additionalfiles1TableS1.docx

- Additionalfiles2Fig.S1.docx

- Additionalfiles3Tables2..docx

- Additionalfiles4Tables3.docx

- Additionalfiles5TableS4.docx

- Additionalfiles6Fig.S2.docx

- Additionalfiles7Fig.S3.docx

- Additionalfiles8Fig.S4.docx

- Additionalfiles9TableS5.docx 